\title{
Las contradicciones de la modernidad y la pregunta por el individuo en las ciencias sociales contemporáneas
}

\author{
Pamela Caro Molina*
}

RESUMEN

En este artículo se abordan perspectivas teóricas sobre las características de la modernidad y su articulación con los debates contemporáneos sobre el individuo en la teoría social. Se afirma que la modernidad avanzada tiene ambigüedades que se expresan en nuevos modos de vida, los que son posibles de conocer a través del tamiz del individuo y de una comprensión crítica y no polarizada.

Palabras clave: Modernidad - teoría social - individuo - riesgo

As contradições da modernidad e a pergunta pelo indivíduo nas ciências sociais contemporâneas

\begin{abstract}
RESUMO
Neste artigo abordam-se perspectivas teóricas sobre as características da modernidad e sua articulación com os debates contemporâneos sobre o indivíduo na teoria social. Afirma-se que a modernidad avançada tem ambigüedades que se expressam em novos modos de vida, os que são possíveis conhecer através do tamiz do indivíduo e e de um entendimento crítico e não polarizada.
\end{abstract}

Palavras-chave: Modernidad - teoria social - indivíduo - risco

The contradictions of modernity and the question of the individual in contemporary social science

\section{ABSTRACT}

This article discusses the features of modernity and its links with contemporary debates about the individual in social theory. One affirms that the advanced modernity has ambiguities which are expressed in new ways of life, which can be known through the sieve of the individual and a critical understanding and not polarized.

Key words: Modernity - social theory - individual - risk 


\section{Introducción}

A través de la revisión y análisis crítico de cinco autores que tratan la temática de los cambios de época y desafíos teóricos, se abordan algunas interrogantes que devienen de las características del proceso de modernidad, del impacto en la teoría social y de la relevancia de la pregunta por el individuo. ¿Cuáles son las características más importantes de la época histórica de la modernidad?, ¿cuáles son los nuevos modos de vida que el proyecto de la modernidad trae?, ¿qué elementos aportan al debate contemporáneo sobre el sujeto e individuo en la teoría social? Los autores revisados son principalmente occidentales (de Europa y Norteamérica) ${ }^{1}$.

La tesis central de esta artículo es que la modernidad como proceso, tiene ambigüedades y contradicciones que se expresan en nuevos modos de vida individuales y sociales, a nivel familiar, laboral y político, lo que ha generado nuevos desafíos para la teoría social, y que no pueden ser mirados como parcelas, sino que requieren ser comprendidos desde las ciencias sociales de manera integrada y dialéctica.

Para desarrollar los argumentos teóricos que respaldan dicha tesis, se presenta una lectura crítica ordenada de los autores, "haciéndolos dialogar", sobre las características y el proceso histórico de la modernidad, las lecturas de cambio de época; las perspectivas de los autores sobre la segunda modernidad que devienen en nuevos desafíos para la teoría social; y las diversas respuestas a la pregunta sobre el sujeto e individuo en las ciencias sociales contemporáneas.

\section{Las paradojas de la modernidad}

Existen diversas entradas a la modernidad; sin embargo, existe coincidencia en dos aspectos aparentemente centrales. Los clarososcuros que representa la modernidad y el carácter transversaluniversal que refleja su radicalización, a partir de la globalización o mundialización.

1 Salvo Danilo Martuccelli, uno de los autores trabajados, quien es peruano, pero que está a cargo de las investigaciones sociológicas en la CNRS-CLERSE de la Escuela de Altos Estudios en Ciencias Sociales de la Universidad de París. http://www.firex21.com/ detalles.aspx?id=9789500371957 
Para Marshall Berman (1985; 1995), filósofo e historiador estadounidense, la paradoja de la modernidad es encontrarnos en un entorno que nos promete aventuras, poder, alegrías, crecimiento, transformación de nosotros y del mundo, pero al mismo tiempo, amenaza con destruir todo lo que tenemos, sabemos y somos (Berman, 1995: 1). Dicha paradoja es transversal, une a toda la humanidad. Los ambientes y experiencias modernas cruzan todas las fronteras de la geografía y la etnicidad, de las clases y la nacionalidad, de la religión y la ideología. Es la paradoja de la desunión, porque "nos arroja a un remolino de desintegración y renovación perpetuas, de conflicto y contradicción, de ambigüedad y angustia" (Berman, 1985:1). En sintonía con la idea de transversalidad y universalidad de la paradoja de la modernidad de Berman, Ulrich Beck, sociólogo alemán, plantea que con la migración transfronteriza se ve la radicalidad de la modernidad. Al mismo tiempo que la idea de biografía ${ }^{2}$ se abre a una sociedad mundial (Beck 2002: 173).

Una de las tesis centrales de Berman es que la modernidad se presenta como una amenaza radical a su historia y tradiciones, pero en el curso de cinco siglos, desarrolló una historia fértil y una tradición propia. Señala la importancia de comprender la historia de esas tradiciones, para entender el modo en que pueden alimentar y enriquecer nuestra propia modernidad, y la forma en que oscurecen o empobrecen nuestra idea de lo que es y puede ser la modernidad (Berman 1985:1).

Antonhy Giddens, sociólogo británico, nombra el momento actual de la modernidad, señalando que estamos en una "desorientación", atrapados en un universo de acontecimientos que no logramos entender del todo y parecen escapar de nuestro control. Por ende no basta con inventar términos como postmodernidad, sino que debemos posar una nueva mirada sobre la naturaleza de la propia modernidad. Al igual que Berman y Beck, Giddens plantea que las consecuencias de la modernidad se están radicalizando y universalizando como nunca (Giddens 1993: 17).

La modernidad en el siglo XX es un fenómeno de doble filo. El desarrollo de las instituciones sociales modernas y su expansión mundial han creado oportunidades enormemente mayores para

2 Que será abordada más adelante en este artículo. 
que los seres humanos disfruten de una existencia más segura y recompensada que cualquier tipo de sistema premoderno. Pero la modernidad tiene también su lado sombrío (Giddens 1993: 20).

Modernidad no es igual a modernización y modernismo. Berman señala que la modernización son los procesos sociales que dan origen a la vorágine de cambios generados por la industrialización de la producción, manteniéndola en un estado de perpetuo devenir. Las personas serían sujetos y objetos de la modernización, en tanto tienen el poder de cambiar el mundo que está cambiándoles, abrirse paso a través de la vorágine y hacerla suya. Por modernismo entiende los valores y visiones de la modernidad (Berman 1995:2).

\section{Historia de la modernidad, vista desde el mundo occidental}

Para situar contextualmente las visiones actuales sobre modernidad es clave revisar el recorrido histórico que hacen los autores trabajados. Existiría coincidencia en señalar que la modernidad como época histórica tiene fases o momentos.

De acuerdo a Berman, la primera fase de la modernidad se da desde comienzos del siglo XVI hasta finales del siglo XVIII. Las personas empiezan a experimentar la vida moderna. Sin embargo, no saben bien con qué se han tropezado, buscan un vocabulario adecuado, no se sienten pertenecer a una comunidad moderna con la cual compartir esfuerzos y esperanzas (Berman, 1995:2). La segunda fase, llamada modernidad ilustrada, comienza con la gran ola revolucionaria de la década de 1790, con la Revolución Francesa y sus repercusiones, surge abrupta y espectacularmente al gran público moderno. Las insurrecciones explosivas son en todas las dimensiones de la vida personal, social y política. En el siglo XIX el público moderno vivía una dicotomía interna, el vivir en dos mundos, en uno que no es moderno material y espiritualmente, pero basado en un discurso moderno (Berman 1995: 3).

La tercera y última fase se ubica en el siglo XX. El proceso de modernización se expande. La cultura del modernismo triunfa en el arte y el pensamiento. Diversos autores coinciden en señalar que a partir de la década de los cincuenta o sesenta estaríamos en presencia de la modernidad avanzada, modernidad tardía o modernidad post- 
industrial, dependiendo del foco analítico (Berman 1995; Taylor 1996; Giddens 1993).

\section{Características de la modernidad avanzada: tres perspectivas de análisis}

Para ordenar el desarrollo argumental, nos centraremos en las preguntas en relación a la modernidad actual y sus vínculos con los modos de organización social, las formas de representación del mundo y la aparición de ciertos modos de vida, desde la perspectiva de tres autores: Berman, Giddens y Taylor.

El retorno a las raíces de la modernidad (Berman): Para Marshall Berman, a medida que el público moderno crece, se expande, se divide en multitud de fragmentos que hablan idiomas extraordinariamente privados, la modernidad pierde gran parte de su vitalidad, resonancia y profundidad, y mucho de su capacidad para organizar y dar sentido a la vida de la gente, teniendo como consecuencia la pérdida del contacto con las raíces de su propia modernidad, que es la voz moderna arquetípica. No sabemos cómo usar nuestro modernismo, hemos roto la conexión entre nuestra cultura y vidas. Cita la novela "La nueva Eloísa" de Rosseau, señalando que ésta inaugura el sentido en que se usará el concepto de modernidad en los siglos XIX y XX. En el sentido de la vida en tensión, experimentar la vida como un torbellino social el protagonista anhela algo sólido a lo que asirse frente al asombro y miedo, todos entran en contradicción consigo mismos, todo es absurdo pero nada chocante, se presenta una multitud de nuevas experiencias, el que quiera gozarlas debe estar dispuesto a cambiar sus principios con su público, ajustar su espíritu en cada paso (Berman 1995: 2- 4). En esta atmósfera nace la sensibilidad moderna.

Para Berman, el modernismo de los sesenta puede dividirse en tres grandes tendencias basadas en las actitudes hacia la vida moderna en su conjunto: afirmativa, negativa y marginada. Son visiones orientadas a las polarizaciones rígidas y totalizaciones burdas, aceptadas ciegamente o condenadas con desprecio, son menos sutiles y dialécticas (Berman 1995: 11-18).

La visión afirmativa, llamados a sí mismos posmodernistas, pensaban que el modernismo de la forma y revolución pura era demasiado 
estrecho, opresivo del espíritu moderno. En el arte insuflaron aire fresco, lúdico; pero no desarrollaron una perspectiva crítica, que clarificara el punto de apertura en que el mundo moderno debía detenerse (Berman 1995: 21).

En el origen del polo de los pesimistas, está el pensamiento de Weber ${ }^{3}$ quien señaló que el poderoso cosmos del orden económico moderno (inexorable, capitalista, legalista y burocrático) es visto como "una jaula de hierro". Para Weber la modernidad no es sólo una jaula, sino que todos los que habitan están configurados por sus barrotes. El hombre moderno como sujeto, ser vivo capaz de respuesta, juicio y acción en y sobre el mundo, ha desaparecido (Berman, 1995: 16). Giddens también menciona el pesimismo de Weber, quien vio el mundo moderno como una paradoja en que el progreso material sólo se obtenía a costa de la expansión de la burocracia que aplastaba la creatividad y autonomía individual (Giddens 1993: 20).

Análisis institucional de la modernidad (Giddens): A fines del siglo XX se plantea el comienzo de una nueva era que trasciende a la misma modernidad. Ha surgido un nuevo tipo de sistema social. El estado anterior de las cosas está llegando a su fin (Giddens 1993: 15).

Coincide en cierto sentido con Berman (en el sentido de las visiones rígidas), al señalar que las tradiciones teóricas clásicas en sociología (Marx, Durkheim, Weber) interpretan la naturaleza de la modernidad fijándose en una única dinámica de transformación, lo que impide un análisis satisfactorio de las instituciones modernas. Para Marx la principal fuerza transformadora del mundo moderno es el capitalismo, por lo que el orden social que emerge de la modernidad es capitalista. Para Durkheim el orden social que emerge es industrial. Para Weber es el capitalismo racional (Giddens 1993: 23-24). Este autor propone que la modernidad es multidimensional en el plano de las instituciones, no puede ser vista por parcelas y que cada uno de los elementos especificados por estas distintas tradiciones desempeña algún papel (Giddens 1993: 24).

Una de sus tesis centrales es que la cuestión del orden es un problema de cómo es que los sistemas sociales cohesionan el tiempo con el espacio (presencia-ausencia). El dinamismo de la modernidad deriva

3 Ver: La Ética Protestante y el espíritu del capitalismo, 1904. 
de la separación del tiempo y del espacio y de su recombinación de tal manera que permita una precisa regionalización de la vida social. Introduce el concepto de desanclaje de los sistemas sociales (Giddens 1993: 28) ${ }^{4}$. La separación tiempo y espacio corta las conexiones que existen entre la actividad social y su anclaje en las particularidades de los contextos de presencia.

Las instituciones sociales modernas mantienen discontinuidades con los órdenes sociales tradicionales en lo que respecta al ritmo de cambio que la era de la modernidad pone en movimiento (celeridad del cambio), el ámbito del cambio (se trata de un cambio globalizado), y la naturaleza intrínseca de las instituciones modernas, por ejemplo el trabajo asalariado (Giddens 1993: 19).

La expansión de la modernidad en el siglo XX coincidió con la organización social del tiempo. El vaciado temporal ${ }^{5}$ es una precondición para el vaciado espacial y tiene prioridad, porque la coordinación a través del tiempo es la base del control del espacio (Giddens 1993: 29).

Para Giddens la radicalidad de la modernidad se expresa en la evaporación de la privilegiada posición de Occidente y en la expansión creciente de las instituciones modernas en todo el mundo. En la interpretación discontinuista, la modernidad no es una civilización entre otras. Aún no vivimos en un universo social posmoderno, pero podemos vislumbrar el surgimiento de modos de vida y formas de organización social que divergen de aquellos

4 Habrían dos mecanismos de desanclaje: a) Las señales simbólicas, por ejemplo el dinero, que pueden ser pasadas de unos a otros sin consideración por las características de los individuos o grupos que los manejan en una particular coyuntura. El dinero sería un medio de distanciamiento entre el tiempo y el espacio, pues permite la verificación de transacciones entre agentes ampliamente separados en tiempo y espacio. El dinero se relaciona con el tiempo como un medio de aunar a ambos al enlazar instantaneidad y aplazamiento, presencia y ausencia (Giddens 1993: 32-35). b) Los sistemas expertos, operados bajo la fiabilidad, apoyada en un conocimiento inductivo débil y en agencias reguladoras (por ejemplo quienes dan licencias). Son sistemas de logros técnicos o de experiencia profesional que organizan grandes áreas del entorno material y social en el que vivimos (Giddens 1993: 37).

5 El invento del reloj mecánico fue crucial en la separación del tiempo del espacio. Expresó una dimensión uniforme del tiempo "vacío" cuantificándolo de tal manera que permitió la designación de horas del día (por ejemplo la jornada laboral). En el mundo premoderno la vinculación del tiempo con el espacio era normalmente imprecisa e invariable. No se podía saber la hora del día sin hacer referencia a otros indicadores socio-espaciales, el cuándo estaba universalmente conectado al dónde (Giddens 1993). 
impulsados por las instituciones modernas (Giddens 1993: 57). En síntesis, la radicalidad y dinamismo de la modernidad, en el sentido de rapidez, como desprendimiento de los órdenes tradicionales, se debe al distanciamiento entre tiempo y espacio, al desanclaje y a la reflexividad. Estas fuentes son condiciones que facilitan las transiciones históricas. Están implicadas las dimensiones institucionales de la modernidad. La mundialización se refiere al proceso de alargamiento en lo concerniente a los métodos de conexión entre diferentes contextos sociales convertidos en una red a lo largo de toda la Tierra (Giddens 1993: 67).

Por ejemplo, el capitalismo, basado en su poder económico más que político, desde un principio fue un asunto de la economía mundial y no de Estados nacionales, determinando sus aspiraciones por fronteras nacionales. Entonces para Giddens las dimensiones o ejes institucionales de la modernidad, que siempre están vinculados, son el Capitalismo, el Industrialismo, la Vigilancia, y el Poder militar (Giddens 1993: 71).

El sentimiento y el yo moderno (Taylor): Este filósofo canadiense pone su foco de análisis sobre los nuevos modos de vida que el proyecto de la modernidad trae, en la génesis del "yo moderno". La noción moderna es la idea del yo desvinculado o capaz de distanciarse de su tradición. Por lo tanto, señala que es necesario escudriñar lo particular para llegar a lo general (Taylor 1996: 304). Las fuentes morales están dentro de nosotros mismos, en una "naturaleza interior que señala lo que es significativo para nosotros" (Taylor 1996: 320).

Su tesis es que el giro deísta abrió el camino para un nuevo papel y sin precedentes del sentimiento, cuya importancia se plasma en toda la sociedad. Entonces, el gran cambio cultural de la identidad moderna es la centralidad del sentimiento: nuevo amor a la naturaleza, culto a la sensibilidad. No es que antes no existiera, sino que ahora comienza a percibirse como parte crucial de lo que hace que una vida sea valiosa y significativa. Como consecuencia, la infancia gana identidad, con sus peculiares sentimientos y necesidades, y la crianza de los hijos se convierte en tema. Es paralelo y está entretejido con la progresiva y amplia difusión de los principios de autonomía y las prácticas de la introspección (Taylor 1996: 303-310).

El sentimiento adquiere relevancia moral y se convierte en elementos 
de legitimidad y en la clave del bien humano. Experimentar ciertos sentimientos pasa a ser parte de la vida buena (entre ellos el amor conyugal y hacia los hijos). Los sentimientos morales se hacen significativos al igual que los sentimientos de benevolencia, y más tarde los sentimientos estéticos (Taylor 1996: 312).

\section{La pregunta por el individuo en la modernidad}

¿Cuáles son los debates contemporáneos sobre el sujeto e individuo en la teoría social? Para Berman (1995), en tiempos modernos el individuo se atreve a individualizarse. Sin embargo, necesita un conjunto de leyes propias, habilidades, astucias para su autoconservación, autoelevación, autodespertar y autoliberación. La promesa del hombre moderno, el sentido de si mismo se convierte en un gusto por probarlo todo, pero ¿cómo encontrarán los recursos para hacer frente a su todo?

En el caso de Taylor (1996), cuando aborda la centralidad del sentimiento en la modernidad, plantea que la nueva comprensión del matrimonio es paralela a una nueva individualización e interiorización, existe una demanda mayor por el compromiso personal y emocional. El acento en la individuación y el compromiso personal coloca en un lugar de mayor importancia el acuerdo contractual.

Danilo Martuccelli (2007) señala que el individuo es el horizonte preliminar de nuestra percepción social, es en referencia a sus experiencias que lo social obtiene o no sentido, pues la sociedad deja de ser la escala de comprensión exclusiva de los fenómenos sociales. El individuo es el tamiz de nuestras percepciones. Plantea que la sociología dispone de tres estrategias para estudiar al individuo: la socialización, la subjetivación y la individuación.

Haciendo una síntesis apretada de las tres vías desarrolladas por el autor, diremos que la socialización es el proceso de fabricación social y psicológica del actor, mediante el cual los individuos se integran a una sociedad. El paso de una sociedad tradicional a una marcada por la diferenciación social, sociedad moderna, con orientación a la autonomía, da mayor importancia al proceso de fabricación del actor. Los individuos se construyen en estrecha relación con las estructuras 
sociales -valores de una cultura, normas de conducta, instituciones, clases sociales y estilos familiares-. La socialización es una forma de programación individual que asegura la reproducción del orden social a través de una armonización de las prácticas y posiciones, gracias a las disposiciones (habitus) (Martuccelli 2007: 20-22).

En la segunda vía, la subjetivación, el individuo se transforma en actor para fabricarse en sujeto. En una modernidad sometida a la expansión continua del proceso de racionalización, la subjetivación asocia la noción de sujeto colectivo a la idea del proyecto de emancipación. Aún vivimos dentro de la estructura analítica de dicha matriz: un principio de dominación (reificación engendrada por el capitalismo) y un proyecto de emancipación organizado en torno a un sujeto colectivo (el proletariado) (Martuccelli 2007: 25-26). En el estudio de la subjetivación aparece la dupla emancipaciónsujeción. La pregunta es: ¿por qué y cómo los individuos pueden ir más allá de la sujeción? La tercera vía, la de la individuación, estudia a los individuos a través de las consecuencias que induce para ellos el despliegue de la modernidad. La pregunta es por el tipo de individuo que fabrica una sociedad. En el caso de sociedades complejas y altamente diferenciadas, se produce un individuo fuertemente singularizado, es el "actor" de la modernidad (Martuccelli 2007: 30-31).

Para Martuccelli la entrada por el individuo, término simple, polisémico, que es un agente empírico y al mismo tiempo un ser moral, es la mejor entrada para comprender a las sociedades actuales. Tiene una ventaja por su claridad analítica, pues todas las sociedades fabrican individuos. Las preguntas que debemos buscar resolver son ¿cuáles son los modos de fabricación de individuos en los procesos de individuación?, ¿cómo en cada sociedad se fabrica a los actores de la modernidad?

Beck (2002), a partir de la mirada sociológica a la Alemania Federal de las últimas dos décadas, sostiene que se estaría en un cambio categorial en la relación entre individuo y sociedad. Señala que "la modernización conduce a una triple individualización. i) La disolución de las precedentes formas sociales históricas y de los vínculos de dependencia (subsistencia y dominio tradicional) -dimensión de liberación- ii) pérdida de estabilidad y de seguridades tradicionales en relación al saber hacer, creencias y normas -dimensión del desencanto- iii) nuevo tipo de cohesión social-dimensión de control 
o de integración- donde el concepto se convierte en su contrario" (Beck 2002: 163).

La pregunta de Beck es cómo concebir la individualización en función del cambio de las condiciones de vida y del modelo biográfico. Para desarrollar su trabajo teórico analiza las siguientes preguntas, ¿cuáles son las condiciones y medios que fomentan la emancipación?, ¿a qué nuevas formas de socialización y control conducen? (Beck 2002: 165).

Plantea tres tesis. La primera, que ya no se produce una integración por la vía de la reproducción social. Las clases sociales ya no cumplen el rol de grupos de estatus, ni el marco estable de "la familia" marca los vínculos sociales de clase. Es el propio individuo el que se convierte en la unidad de reproducción vital de lo social (Beck 2002: 166). La segunda tesis es que los medios que ocasionan una individualización determinan a su vez una estandarización. Las condiciones individuales originadas son cada vez más dependientes del mercado (cita a Simmel, quien mostró cómo el dinero individualiza y estandariza) y del trabajo productivo, "se trata de la flexibilización del trabajo remunerado y la descentralización del lugar de trabajo, por ejemplo el teletrabajo" (Beck 2002: 167). La tercera tesis señala que la simultaneidad de individualización, institucionalización y estandarización no da cuenta suficiente de las condiciones individuales originadas. Son condiciones individuales dependientes de las instituciones. La individualización se convierte en la forma más avanzada de socialización dependiente del mercado, de las leyes y de la educación (Beck 2002: 167). Sin embargo, en los procesos de individualización no desaparecen las diferencias de clase ni las relaciones familiares, sino que permanecen en el transfondo del modelo biográfico vital. Para Beck, en la modernidad avanzada se realiza la individualización, que significa institucionalización y con ello, configuración política de la vida y de las condiciones de vida, bajo las condiciones de un proceso de socialización que hace casi imposible la existencia autónoma e independiente. El individuo rompe los lazos tradicionales y las relaciones de protección (clase social, familia), pero los intercambia por constricciones del mercado de trabajo y del consumo, así como por la estandarización y control de dichas constricciones (Beck 2002: 167-170).

La sociedad nacida de las formas de vida de la sociedad industrial (clases sociales, familia nuclear, funciones sexuales y oficio) se 
enmarca en un sistema institucional de una época industrial que se extingue. Las instituciones intervienen en la vida "desviada". Formula la pregunta sobre cómo en una sociedad individualizada se concibe el destino colectivo prefigurado institucionalmente y vinculado a la vida. La individualización y privatización (separación de los lazos sociales) genera un doble proceso. Por un lado, formas de percepción privadas y ahistóricas (por ejemplo los hijos ignoran la situación de sus padres), todo gira en torno al propio yo de la propia vida. Por otro lado, la propia vida se configura como el mero producto de las circunstancias (disminuyen ámbitos de cooperación). La biografía personal queda fuera de pautas y abierta a elegir, son autoproducidas, autoelaboradas, autoplanificadas, pero en un contexto de constricción institucional.

\section{El riesgo y la inseguridad en la vida personal y social}

En los textos de Giddens y especialmente en Beck, es posible configurar el abordaje de la noción de riesgo. Giddens señala que dicha noción aparece sólo en el período moderno, al mismo tiempo que la idea de azar. Fiabilidad supone conocimiento de las circunstancias del riesgo (por el conocimiento experto y las agencias reguladoras). Presupone el peligro, pero no el conocimiento del peligro mismo. Frente a los ambientes de riesgo, la seguridad sería definida como la situación en la que los peligros quedan contrarrestados o minimizados (Giddens 1993: 44).

Beck señala que estamos en una sociedad del riesgo (a partir del caso alemán, pero del que es posible hacer extrapolaciones para el caso chileno o latinoamericano). La dependencia de las instituciones incrementa las situaciones de crisis de las condiciones individuales. Para el autor, la clave de la seguridad en la vida reside en el mercado de trabajo (Beck 2002: 170), por ende podría plantearnos que también la clave de la inseguridad. Las instituciones actúan con categorías fijas de biografías normales que cada vez son menos reales, por ejemplo respecto de cómo funciona el mercado de trabajo en la actualidad y cómo se calcula la previsión social. Ya no se puede calcular en base a un trabajo estable, continuo, que ha dejado de existir en el modelo de flexibilidad. 
Con la autoelaboración de las biografías en la sociedad individualizada, los riesgos aumentan y surgen nuevas formas de culpabilización, lo que representa una sobrecarga (Beck 2002: 173). Frente a las constricciones que imponen las instituciones y la estandarización surge el conflicto y el riesgo que impiden toda solución individual. Se agudizan las oposiciones entre la normalidad que rige a las instituciones y la propia sociedad individualizada, la que se ve atravesada por conflictos, vínculos y fronteras (Beck 2002: 171).

Si tomamos la perspectiva teórica de Beck podemos ver que uno de los rasgos distintivos del empleo, en la actualidad, en el caso de Chile, es la 'desestandarización' de la jornada laboral normal y la progresiva instalación de múltiples formas de organización del tiempo de trabajo. Las modalidades horarias emergentes perturban las posibilidades de vida familiar compartida con la pareja y/o hijos y constituye un factor de inseguridad. En especial para las mujeres, dado el mandato tradicional de ser las responsables principales del cuidado de los hijos/as. Esto, porque paralelo a que se integran en forma creciente al mercado laboral, como una forma de responder a una aspiración propia de acceder a un derecho que les brinda autonomía y a la necesidad de sostener una vida familiar en condiciones de mayor calidad, siguen siendo las responsables del trabajo doméstico (Todaro 2004) y de cuidado. Bajo este marco de análisis, hombres y especialmente mujeres que se enfrentan a las tensiones del trabajo productivo y reproductivo de cuidado estarían experimentando el riesgo como consecuencia de la radicalización de la modernidad.

\section{Conclusiones}

¿Cuáles son las principales contradicciones de la actual modernidad?, ¿cómo podemos verlas a través del tamiz del individuo y del sujeto?, son las interrogantes que emergen al iniciar las conclusiones articuladas en relación a la tesis central de este trabajo. Definitivamente Berman nos ayuda a dilucidar aquellas contradicciones. A través de sus referencias a los modernistas del siglo XIX, Marx y Nietzche, quienes captaron las contradicciones de la modernidad con todas sus fuerzas, en todos los momentos de su vida diaria, nos plantea finalmente que la "promesa de la modernidad" trae peligros, 
dolor y miedo ${ }^{6}$; pero al mismo tiempo ambos autores creían en la capacidad de salir adelante. Berman plantea que posando la mirada en las raíces de la modernidad aparece lo contradictorio y dialéctico, que es denunciar la vida moderna en nombre de los valores que la propia modernidad ha creado (Berman 1995: 10).

Concluye que lo fundamental de la vida moderna es su radicalidad contradictoria de base. Por ejemplo, la máquina cuya propiedad maravillosa acorta y hace más fructífero el trabajo humano, provoca al mismo tiempo el hambre y agotamiento del trabajador. Las fuentes de riqueza se convierten en fuentes de privaciones. El dominio del hombre de la naturaleza es cada vez mayor, pero el hombre se convierte en esclavo de otros hombres y de su propia infamia. Sin embargo, es una contradicción que pudo haber tenido salida y que en la actualidad la puede seguir teniendo. Retoma la fe paradigmáticamente modernista de Marx "para hacer trabajar bien a las nuevas fuerzas de la sociedad se necesita únicamente que éstas pasen a manos de hombres nuevos: los obreros". Por lo tanto, una clase de hombres nuevos, modernos, será capaz de resolver las contradicciones de la modernidad -de superar las presiones aplastantes, y los abismos personales y sociales- (Berman 1995: 6).

Con el análisis planteado como telón de fondo, y a la luz de la devaluación del trabajo y la pérdida de soporte actual para la conformación de este "obrero u obrera nuevo/a", me pregunto: ¿quiénes son y cómo serán esos hombres y mujeres nuevos de la sociedad moderna contemporánea?, ¿(son) serán los ambientalistas, las mujeres (en su doble rol productivo/reproductivo), los movimientos indígenas del Tercer Mundo?, ¿(son) serán capaces de superar las presiones aplastantes, y los abismos personales y sociales?

Giddens por su parte ve las contradicciones y ambigüedades de la modernidad, abordando sus consecuencias. Señala que si bien las instituciones sociales modernas y su expansión mundial han creado oportunidades respecto del sistema premoderno, la modernidad tiene su lado oscuro, que no fue previsto en toda su amplitud.

Frente a las contradicciones y consecuencias de la modernidad, aparece la perspectiva optimista de Berman, sobre el retorno a las raíces de la modernidad, para aprender de ella y nutrir positivamente

6 Así como también inseguridades y riesgos. Beck, 2002. 
los actuales avatares de la vida moderna. En definitiva convoca a observar nuestro propio entorno, con nuevos ojos, volviéndonos a conectar con una cultura modernista rica y vibrante; sintiéndonos comunidad con personas de todo el mundo que luchan con dilemas similares (Berman 1995: 27). Cita a Octavio Paz reforzando el que los modernismos del pasado pueden ayudarnos a asociar nuestras vidas con las de personas que viven el trauma de la modernización en otras sociedades distantes en kilómetros y en tiempo; iluminar el deseo de estar arraigados en un pasado social y personal estable y coherente (Berman 1995: 26).

Berman dice que los científicos sociales han dividido a la modernidad en una serie de componentes separados (industrialización, construcción del Estado, urbanización, desarrollo de los mercados, formación de una elite u otros) y se han opuesto a integrarlos en un todo. Gidenns también alerta sobre el error de analizar a la modernidad y a sus instituciones por parcelas.

Coincidiendo con dichos planteamientos, cabe mencionar lo insatisfactorio que resulta abordar, desde los estudios sociales, por separado los componentes de la modernidad (Berman) o las instituciones de la modernidad (Giddens). Cuestión que cobra un alto sentido, por ejemplo, en los estudios de género, siendo relevante afrontar temas como trabajo y familia, reproducción/producción y otros, desde una mirada dialéctica y holística, que permita comprender la sociedad, sus contradicciones y ambigüedades.

¿Las contradicciones de la modernidad avanzada tienen similares consecuencias para hombres y mujeres en la sociedad contemporánea? Pareciera que no, que es necesario mirar comprensivamente las consecuencias singulares. En el caso de las mujeres chilenas es posible vislumbrar los claro-oscuros que trae la modernidad. La promesa de la incorporación creciente al mercado de trabajo productivo asalariado, y por ende al dinero (que conllevaría a autonomía económica y, de acuerdo a Beck, a la dimensión de "liberación" de la individualización que trae la modernidad), ha resultado cargada de tensiones y conflictos en el ámbito privado (y público), pues se insertan a un trabajo asalariado cuando éste se precariza y devalúa, en un contexto laboral flexible, que ha tenido como consecuencia en las mujeres el surgimiento de "peligros, miedos y dolores". En definitiva vivir la sociedad del riesgo, a partir de la autoelaboración 
(pero sin muchas alternativas) por las constricciones que imponen las formas de operar de instituciones actuales como el mercado de trabajo asalariado. Beck plantea que las intervenciones institucionales son intervenciones en la vida humana. Por ejemplo, la implantación de horarios en los jardines infantiles, como intervenciones institucionales, pueden facilitar o dificultar compaginar los deberes profesionales y maternos (Beck 2002: 168).

Retomando la tesis original, podemos decir que los autores trabajados nos ayudan a comprender las ambigüedades y contradicciones de la modernidad avanzada, que se expresan en nuevos modos de vida en la sociedad contemporánea, los que son posibles que mirar o conocer a través del tamiz del individuo. Parafraseando a Martuccelli, el individuo es el horizonte preliminar de nuestra percepción social, en referencia a sus experiencias lo social obtiene o no sentido. Las consecuencias de la modernidad, en los planos individual y social, en espacios como la familia, el trabajo, la política y otros, han generado y seguirán generando en las ciencias sociales, nuevos desafíos para la teoría social, que implicará seguir haciéndonos preguntas sobre el individuo, la autonomía, el lazo social, entre otras, para lo cual se requiere una comprensión societal crítica, menos rígida y menos polarizada.

\section{Referencias bibliográficas}

Beck, U. (2002). La sociedad del riesgo. Hacia una nueva modernidad. Barcelona: Paidós.

Berman, M. (1985). Brindis por la modernidad. Revista mexicana Nexos. 89

Berman, M. (1995). Todo lo sólido se desvanece en el aire. La experiencia de la modernidad. México: Siglo XXI.

Giddens, A. (1993). Consecuencias de la modernidad. Madrid: Alianza

Martuccelli, D. (2007). Las tres vías del individuo sociológico. Cambio de Rumbo. Santiago, Chile: LOM.

Taylor, C. (1996). Las fuentes del yo. Barcelona: Paidós.

Todaro, R. y Yáñez, S. (2004). El trabajo se transforma. Relaciones de producción y relaciones de género. Santiago, Chile: CEM Ediciones. 